\title{
Interpretation of magnetic profiles using a decomposition in Hilbert transform pairs
}

Saulo Pomponet Oliveira*1, Jeferson de Souza ${ }^{2}$, Francisco José Fonseca Ferreira ${ }^{1}$, and Luis Gustavo de Castro ${ }^{1}$, ${ }^{1}$ Federal University of Paraná, ${ }^{2}$ Paraná State Secretary of Education.

Copyright 2021, SBGf - Sociedade Brasileira de Geofísica.

This paper was prepared for presentation at the $17^{\text {th }}$ International Congress of the Brazilian Geophysical Society, held in Rio de Janeiro, Brazil, August 16-19, 2021.

Contents of this paper were reviewed by the Technical Committee of the $17^{\text {th }}$ International Congress of the Brazilian Geophysical Society and do not necessarily represent any position of the SBGf, its officers or members. Electronic reproduction or storage of any part of this paper for commercial purposes without the written consent of The Brazilian Geophysical Society is prohibited.

\section{Abstract}

We propose a simple transformation to aid the interpretation of magnetic anomalies generated by linear structures. The profile of such anomalies across the strike can be decomposed into two signals, one being symmetric and the other antisymmetric with respect to the center of the source. The symmetric component serves as input data to a variety of depth estimation techniques that often assume the anomaly is reduced to the pole. We use the fact that these components form a Hilbert transform pair to transform a skewed anomaly profile into a symmetric one. Unlike in previous works that rely on the decomposition into even and odd functions, the profile does not need to be shifted to the source's center of symmetry or to be limited to one isolated anomaly. Multiple effective magnetization directions presented by different dikes are modeled by a function representing the different local effective dip angles. We validate the method with synthetic data and ground magnetic survey data from a dike swarm located at Ponta Grossa Arch, southern Brazil. We also illustrate the usefulness of reconstructed anomalies in depth estimation methods. The results show also that the method is capable of handling interfering sources with distinct effective magnetization directions.

\section{Introduction}

The seminal work of Nabighian (1972) on the analysis of two-dimensional structures with the analytic signal is the starting point of numerous enhancement and depth estimation techniques.

The magnetic anomaly profile of a dike model of infinite depth extent, as well as other $2 \mathrm{D}$ structures, can be mathematically expressed in the form $M(x)=A\left[\cos Q f^{s}(x)+\right.$ $\sin Q f^{a}(x)$, where $A$ is an amplitude coefficient and $Q$ is an effective angle that depends on geological and magnetic dips, while $f^{s}(x)$ and $f^{a}(x)$ are composite functions of inverse tangents and logarithms, respectively (Nabighian, 1972). The use of the analytic signal to interpret magnetic data was motivated by the fact that horizontal and vertical derivatives of $M(x)$ constitute a Hilbert transform pair. The Hilbert transform is itself a common interpretation tool (Ram Babu and Atchuta Rao, 1991; Sundararajan et al., 1998). On the other hand, the fact that $f^{s}(x)$ and $f^{a}(x)$ also constitute a Hilbert transform pair has been poorly explored in the literature, to our knowledge.
Hutchison (1958) pioneered the decomposition of magnetic profiles into $f^{s}(x)$ and $f^{a}(x)$, studying their symmetry properties and determining source parameters from their representation in logarithmic scale. In particular, $f^{s}(x)$ and $f^{a}(x)$ become respectively even and odd functions when the origin is shifted to the source center. He also pointed out that these components can be separated by a graphical procedure that was later formalized as a decomposition into even and odd functions (Rao and Murthy, 1967), while the location of the symmetry center was studied by Powell (1967). Bhimasankaram et al. (1978) applied this parity decomposition to obtain the depth, half-width, and the effective angle $Q$ in frequency domain. Kara et al. (1996) have further developed several interpretation tools starting from the same principle. de Souza et al. (2020) proposed a weighted average based on both even and odd components as an alternative to the reduction-to-the-pole filter.

We use the relationship between $f^{s}(x)$ and $f^{a}(x)$ through Hilbert transform to obtain a reconstructed symmetric anomaly without having to displace the origin of the coordinate system to the dike's center. This approach is able to generalize the above studies to the case of multiple dikes and other two-dimensional structures. As pointed out by de Souza et al. (2020), the reconstructed anomaly is useful in depth estimation techniques that have been developed for anomalies with vertical magnetization. Similarly to Paine et al. (2001) and Pilkington and Beiki (2013), the reconstruction algorithm can be employed in an inversion code to reduce the influence of remanent magnetism.

\section{Theory}

Let us consider the magnetic anomaly $M(x, z)=$ $A\left[\cos Q f^{s}\left(x-x_{0}, z\right)+\sin Q f^{a}\left(x-x_{0}, z\right)\right]$,

$$
\begin{aligned}
& f^{s}(x, z)=\tan ^{-1} \frac{x+a}{z_{0}-z}-\tan ^{-1} \frac{x-a}{z_{0}-z} \\
& f^{a}(x, z)=\frac{1}{2} \ln \frac{(x+a)^{2}+\left(z_{0}-z\right)^{2}}{(x-a)^{2}+\left(z_{0}-z\right)^{2}}
\end{aligned}
$$

produced by an infinite dyke model with half-width $a$, depth to top $z_{0}$, and center at $x_{0}$. The effective dip angle $Q$ and amplitude $A$ depend on the geologic dip angle and induced/remanent magnetization. A similar formula applies to finite steps (Nabighian, 1972; Ram Babu and Atchuta Rao, 1991).

For simplicity, we consider the observation height $z=0$ and denote $M(x, 0)=M(x), f^{s}(x, 0)=f^{s}(x)$, and $f^{a}(x, 0)=f^{a}(x)$. As pointed out by Ram Babu and Atchuta Rao (1991), the Hilbert transform of $M(x)$ is $H[M(x)]=-\cos Q f^{a}(x)+$ $\sin Q f^{s}(x)$, thus $H\left[f^{s}(x)\right]=-f^{a}(x)$ and $H\left[f^{a}(x)\right]=f^{s}(x)$, i.e., $f^{s}$ and $f^{a}$ constitute a Hilbert transform pair. It follows that 
the anomaly $M(x)$ and its symmetric component $M^{S}(x)=$ $A f^{a}(x)$ satisfy

$$
\left\{\begin{aligned}
M(x) & =\cos Q M^{s}(x)-\sin Q H\left[M^{s}(x)\right] \\
H[M(x)] & =\cos Q H\left[M^{s}(x)\right]+\sin Q M^{s}(x)
\end{aligned}\right.
$$

Solving the above system for $\left[M^{s}(x), H\left[M^{s}(x)\right]\right]^{T}$, we find

$$
\left[\begin{array}{l}
M^{s}(x) \\
H\left[M^{s}(x)\right]
\end{array}\right]=\left[\begin{array}{ll}
\cos Q & \sin Q \\
-\sin Q & \cos Q
\end{array}\right]\left[\begin{array}{l}
M(x) \\
H[M(x)]
\end{array}\right] .
$$

Note that the components of the zero-order analytic signal (Cooper, 2015) of $M(x)$ and $M^{s}(x)$ are related by a classical rotation matrix with angle $Q$, which does not change the amplitude of the rotated signal, hence the transformation from $M$ to $M^{s}$ is energy-preserving.

The symmetric anomaly $M^{s}(x)$ can be obtained from the first row of equation 3 :

$$
M^{s}(x)=\cos Q M(x)+\sin Q H[M(x)],
$$

noting that the Hilbert transform can be readily computed in Fourier space (Nabighian, 1972). The same procedure can be applied to the spatial derivatives of the total field anomaly, since the derivatives of $f^{s}$ and $f^{a}$ are also Hilbert transform pairs.

As Murthy (1985), we evaluate the effective angle $Q$ as the ratio

$$
Q=\tan ^{-1} \frac{M_{x}\left(x_{0}\right)}{M_{z}\left(x_{0}\right)},
$$

where $x_{0}$ is estimated by the maximum analytic signal amplitude (ASA) (de Souza et al., 2020). In the case of multiple dikes, we interpolate the effective angles at each dike center estimated through equation 5. Moreover, we use a selection criterion based on the threshold peak prominence to locate the most physically significant structures, avoiding spurious peaks generated by noisy data (Pirok et al., 2018).

\section{Results and discussion}

In the following we illustrate the reconstruction formula 4, which we refer to as Hilbert Transform Decomposition (HTD), with examples of synthetic and field anomalies generated by multiple dike-like structures. We obtain reconstructed profiles of the total field anomaly (TFA) as well as its vertical derivative (VDR), and use them to compute source depths. For field data, we also compare the HTD anomalies with those obtained by reduction-tothe-pole (RTP).

\section{Synthetic example}

Let us consider a model of two dikes with half-width $a=20$ $\mathrm{m}$, depths $z_{1}=100 \mathrm{~m}$ and $z_{2}=50 \mathrm{~m}$, centers at $x_{1}=700 \mathrm{~m}$ and $x_{2}=1200 \mathrm{~m}$, and effective angles $Q_{1}=30^{\circ}$ and $Q_{2}=$ $-60^{\circ}$. The TFA is given as $M(x)=M_{1}(x)+M_{2}(x)$, where, for $i=1,2, M_{i}(x)=A\left[\cos Q_{i} f_{i}^{s}\left(x-x_{i}\right)+\sin Q_{i} f_{i}^{a}\left(x-x_{i}\right)\right]$, with $A=200 \mathrm{nT}$, and

$$
\begin{aligned}
f_{i}^{s}(x) & =\tan ^{-1} \frac{x+a}{z_{i}}-\tan ^{-1} \frac{x-a}{z_{i}}, \\
f_{i}^{a}(x) & =\frac{1}{2} \ln \frac{(x+a)^{2}+z_{i}^{2}}{(x-a)^{2}+z_{i}^{2}} .
\end{aligned}
$$

We added Gaussian noise to TFA with a standard deviation of $0.1 \mathrm{nT}$. In Figure 1 we present the noise-corrupted TFA (Fig. 1a) along with its VDR (Fig. 1b). Figure 2a shows the analytic signal amplitude (ASA), where the peaks with threshold prominence of $10 \%$ are highlighted.
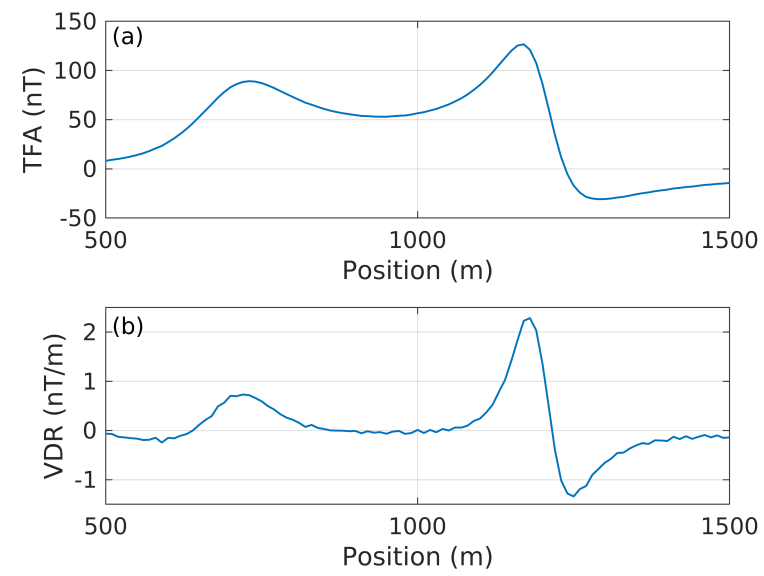

Figure 1: (a) Total field anomaly (TFA) generated by two dikes. (b) Vertical derivative (VDR) of the data in (a).

The function representing the effective dip angle (Fig. 2b) is constant over each individual anomaly and equal $Q_{i}=$ $\tan ^{-1} M_{x}\left(x_{i}\right) / M_{z}\left(x_{i}\right)$, which is estimated from equation 5 using the anomaly source center $x_{i}$. The domain of each anomaly lies between the two minima of ASA (squares in Figure 2a) adjacent to the maximum in $x_{i}$. We connect these constant functions by linear interpolation, as shown in Figure 2b.
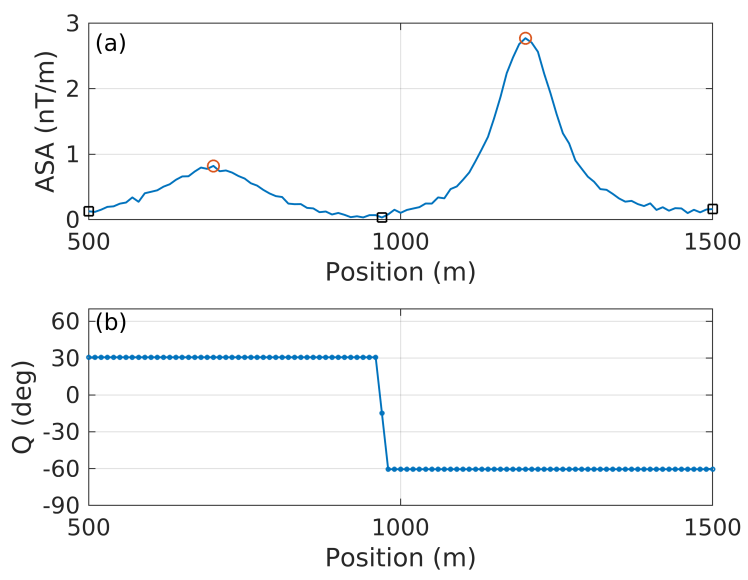

Figure 2: (a) ASA of the data in Figure 1a. Circles and squares denote relative maxima and minima of ASA, respectively, with threshold peak prominence of $10 \%$ of the absolute maximum. (b) Interpolated effective dip angle. Each plateau represents the effective dip angle of an individual dike and the gap between them is located over a minimum of the ASA.

The HTD reconstruction of TFA may produce a discontinuity at the transition between dikes depending on the difference in the magnitude of adjacent effective 
angles. In this case, one should split the profile or conform the base level of each dike so that their anomalies match at the average level (Fig. 3). We have noted from the numerical experiments that such a correction is not necessary on the reconstructed symmetric part of the anomaly derivatives.

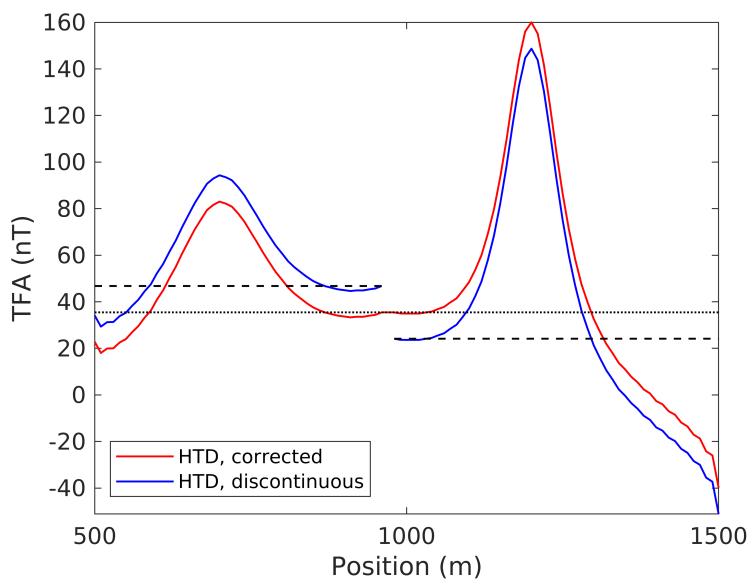

Figure 3: The steep variation in the estimated effective dip angle leads to a discontinuity in the reconstructed anomaly (blue). By adjusting the base level of the contribution from each dike (dashed lines) to their average (dotted line), we obtain a continuous reconstructed anomaly (red).

In Figure $4 \mathrm{a}$, the corrected reconstruction of TFA is compared with the noise-free theoretical one, $M^{s}(x)=$ $A\left[f_{0}^{s}\left(x-x_{0}\right)+f_{1}^{s}\left(x-x_{1}\right)\right]$, while the HTD reconstruction of VDR and $M_{z}^{s}$ are shown in Figure $4 \mathrm{~b}$. The reconstructed symmetric anomaly is significantly affected by the truncation of the Hilbert transform numerical calculation. On the other hand, the reconstructed VDR fits very well the theoretical one, except at the transition between the dikes. Nevertheless, the gap between the individual anomalies is barely visible.
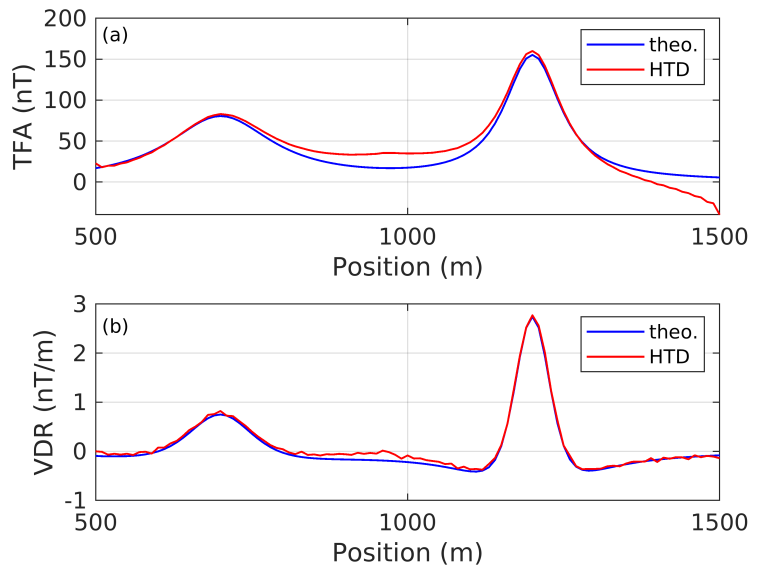

Figure 4: Reconstruction of the magnetic profile shown in Figure 1a, compared with the noise-free symmetric part of the anomaly (Theo), $M^{s}(x)=A\left[f_{0}^{s}\left(x-x_{0}\right)+f_{1}^{s}\left(x-x_{1}\right)\right]$. (a) HTD reconstruction of the TFA. (b) HTD reconstruction of the VDR.

\section{Field example}

Our study area is located in the Ponta Grossa Arch (PGA), southern Brazil. A major feature of the PGA structural framework, presented by Ferreira (1982), is the presence of four magnetic lineaments, namely Guapiara (northern limit), São Jerônimo-Curiúva and Rio Alonzo (central region), and Rio Piquiri (southern limit), as indicated in Figure 5. These lineaments extend over $600 \mathrm{~km}$ in the NWSE direction and are related to diabase dike swarms.

We use a profile obtained from the ground magnetic survey carried out by Castro et al. (2008). The profile is $12224 \mathrm{~m}$ long with sampling interval of approximately $25 \mathrm{~m}$. The geological map of the Ponta Grossa Arch and the location of the magnetic profile are indicated in Figure 5.

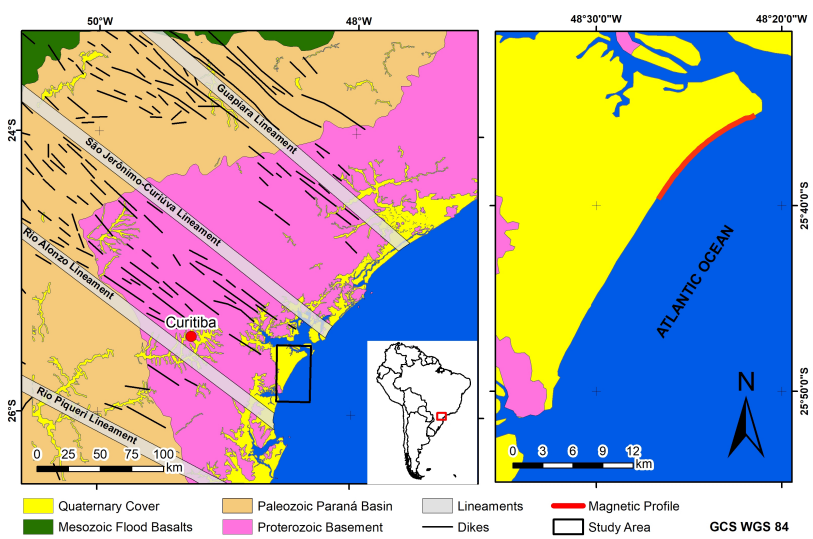

Figure 5: Geological map of the Ponta Grossa Arch with the location of the study area (left) and the magnetic profile (right). Adapted from Louro et al. (2019).

The profile data is shown in Figure 6a, while its VDR is in Figure $6 \mathrm{~b}$. As the synthetic example, we compute the ASA and the effective dip angle (Figs. $7 \mathrm{a}$ and $7 \mathrm{~b}$, respectively).
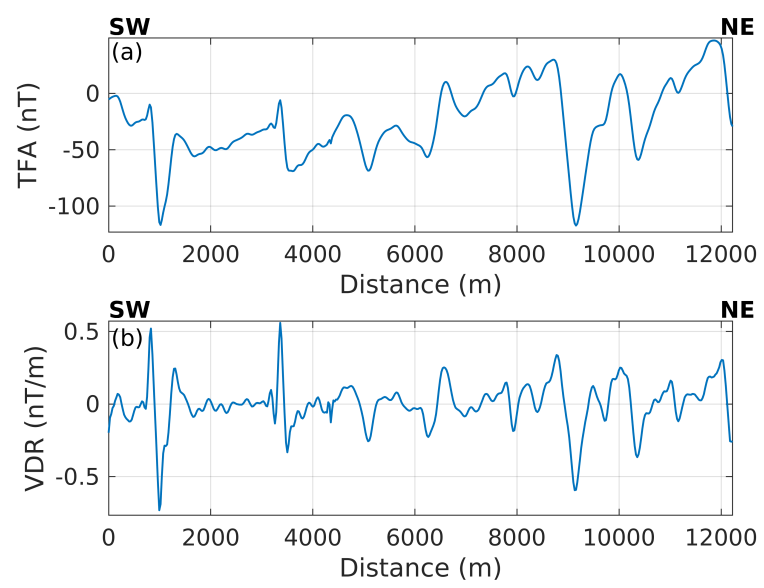

Figure 6: (a) TFA of the magnetic profile shown in Figure 5. (b) VDR of the data in (a).

Figure 8 compares the results of HTD with those obtained through RTP, considering that during the acquisition the inclination and declination of the IGRF field were $-35^{\circ}$ and $-19^{\circ}$, respectively (Castro et al., 2008). Figure $8 \mathrm{a}$ compares HTD and RTP transformations of TFA, while the 

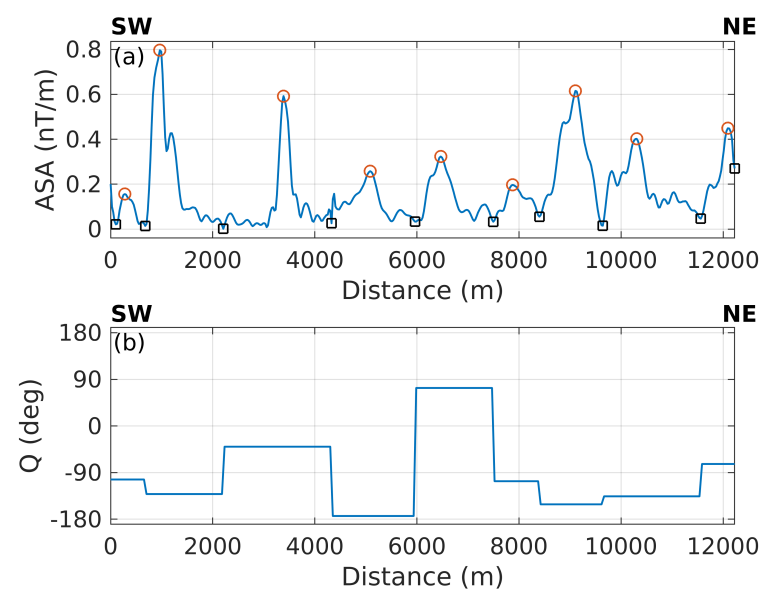

Figure 7: (a) ASA of the data in Figure 6a. Circles and squares denote relative maxima and minima of ASA, respectively, with threshold peak prominence of $10 \%$ of the absolute maximum. (b) Interpolated effective dip angle.

comparisons of VDR are done in separate figures (Figs. 8b and $8 \mathrm{c}$ ). To assess the independence from the effective magnetization direction, the ASA is displayed with the vertical derivatives.

In general, the reconstructed curves are in agreement with RTP anomalies when remanent magnetization is not significant or has a direction similar to the induced one but the former is able to correct the dipolarity due to remanence and/or geological dip while RTP is not capable to. The identification of the remanent magnetization using RTP could be done in combination with other processing techniques in an iterative manner (Roest and Pilkington, 1993). Regarding vertical derivatives, most of the higheramplitude anomaly peaks of the HTD curve coincide with those of the ASA curve (Fig. 8b). Moreover, HTD provides a better resolution of the small-amplitude anomalies than ASA. On the other hand, some peaks of the RTP anomalies are laterally displaced with respect to ASA (Fig. 8c), indicating the presence of significant remanent magnetization in the sources of these anomalies.

\section{Application to depth estimation}

In the following we use the reconstructed anomalies to estimate depth of the dikes using, for comparison, two different methods: Euler deconvolution (Thompson, 1982) and the curvature method (Phillips et al., 2007).

Euler deconvolution for profile data consists in finding the coordinates $\left(x_{0}, z_{0}\right)$ of the source center and a regional field $B$ corresponding to the least-squares solution of

$$
\left(x-x_{0}\right) M_{x}+\left(z-z_{0}\right) M_{z}=N(B-M), \quad x \in W,
$$

where $W=\left\{x_{1}, \ldots, x_{N W}\right\}$ is a moving window that scans the grid. The Euler deconvolution algorithm does not require symmetric anomalies for the dike model (Reid et al., 1990), thus it may be applied either to the original profile or to its HTD reconstruction. We use the structural index $N=1$ and a moving window of $N W=10$ grid points. Moreover, we select the solutions that satisfy the acceptance criterion $z_{0} /\left(N \sigma_{z_{0}}\right) \geq 20$ (Thompson, 1982).
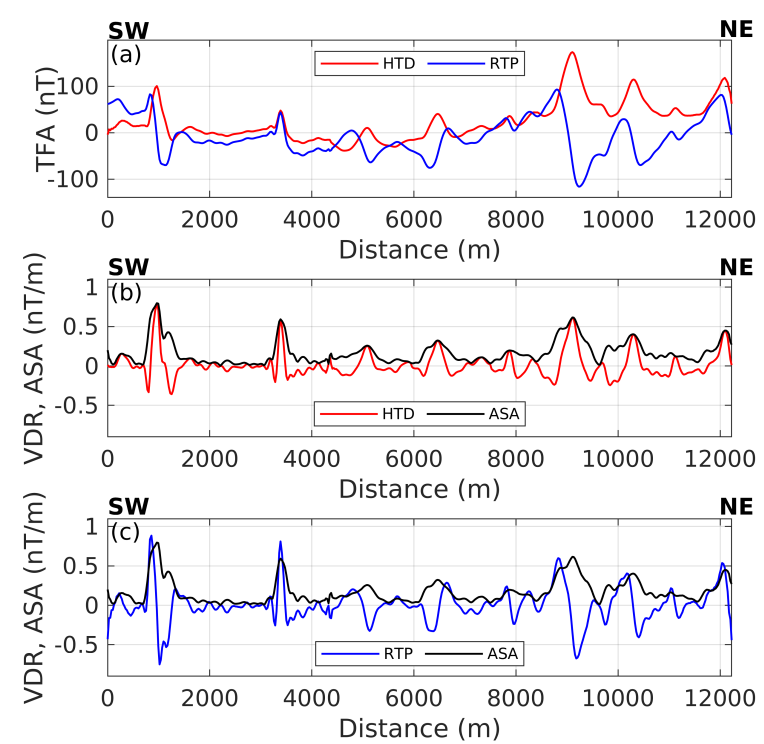

Figure 8: Reconstruction of the magnetic profile shown in Figure 5, compared with the RTP profile. (a) TFA (HTD and RTP). (b) HTD reconstruction of the VDR. (c) VDR of the RTP profile. In (b) and (c), the ASA (black line) is also shown.

In the curvature method, the depth is estimated by the formula

$$
z_{0}\left(x_{0}\right)=\sqrt{-\frac{2 \beta S\left(x_{0}, 0\right)}{K\left(x_{0}, 0\right)}},
$$

where $S(x, z)$ is a transformed anomaly that peaks directly over a source center $\left(x_{0}\right)$ and has the form $S(x, z)=\alpha((x-$ $\left.\left.x_{0}\right)^{2}+\left(z-z_{0}\right)^{2}\right)^{-\beta}$. Phillips et al. (2007) refer to $S$ as the special function. Moreover, $K$ is the curvature of $S$, defined as follows:

$$
K(x, z)=\frac{S_{x x}(x, z)}{\left[1+S_{x x}^{2}(x, z)\right]^{3 / 2}}, \quad S_{x x}(x, z)=\frac{\partial^{2} S}{\partial x^{2}}(x, z) .
$$

Some transformations have been proposed to generate special functions, such as RTP and ASA. We propose obtaining the special function $S$ through HTD reconstruction. Given the analogy between the parameter $\beta$ and the structural index $N$, we use $\beta=1$. Moreover, we compute the second derivatives of $S$ in the curvature, equation 9, using the reconstructed VDR.

Figure $9 \mathrm{a}$ shows the depth estimates by Euler deconvolution using the original (TFA) and the reconstructed (HTD) anomalies. The differences between the estimates using the original or the reconstructed anomaly are not significant, which further confirms that the HTD reconstruction is eligible to use in depth estimation. Figure $9 \mathrm{~b}$ presents the depth estimates provided by the curvature method when the special function $S$ is either ASA or HTD. The solution obtained by ASA is strongly affected by noise in the curvature $K$ (Fig. 9c). The curvature of ASA around the deeper dike has about the same magnitude as the noise, which may explain its lower accuracy for this kind of source. On the other hand, the curvatures of both 
HTD and ASA reach a relatively higher magnitude around the shallower dike, and their depth estimates have nearly the same accuracy. We remark that Phillips et al. (2007) recommended combining ASA with vertical integration to mitigate the noise due to high-order derivatives.
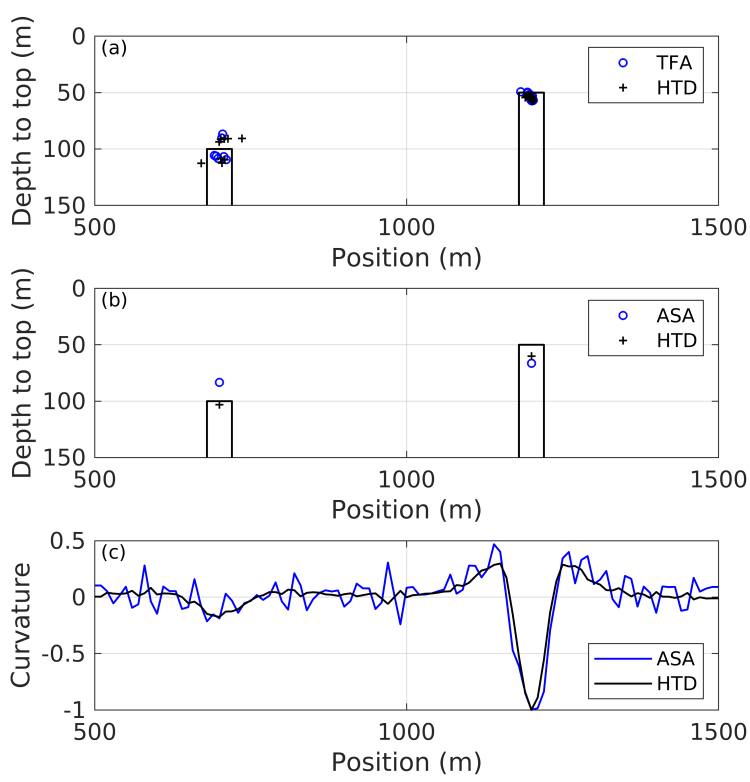

Figure 9: Depth estimates for synthetic data: (a) Euler deconvolution using the original (TFA) and reconstructed (HTD) profiles; (b) Curvature method with special function given by ASA and HTD; (c) Normalized curvature of the special functions.

For the field data, we also consider the depth estimates obtained by RTP. Figure 10 shows the results from Euler deconvolution. The solutions obtained by TFA (Fig. 10a) are less clustered than those based on HTD and RTP (Figs. 10b and 10c), especially around $9000 \mathrm{~m}$ distance. Nevertheless, the distribution of solutions on all approaches are similar to each other, corroborating the low sensitivity of Euler deconvolution to the magnetization direction.

For the curvature method, the results from the ASA special function (Fig. 11a) are less affected by noise than in the synthetic example. These results are similar to the ones from HTD (Fig. 11b) and are concordant with the Euler deconvolution estimates. In both methods, the estimated depths are below $100 \mathrm{~m}$. These estimates agree with the average thickness of sediment layers from three boreholes, located near the study area, that reached the top of the basement (Lessa et al., 2000; Castro et al., 2018). On the other hand, the solutions generated by RTP (Fig. 11c) are significantly discrepant as a result of remanent magnetization.
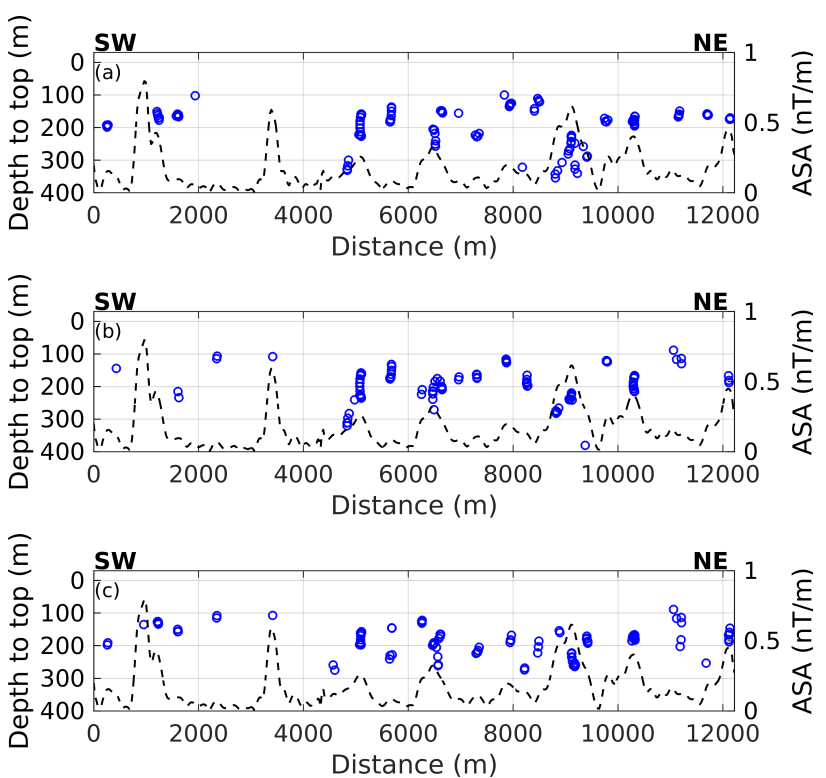

Figure 10: Euler deconvolution solutions obtained for field data using (a) TFA (data from Fig. 6a), (b) HTD (data from Fig. 8a, red), and (c) RTP (data from Fig. 8a, blue).
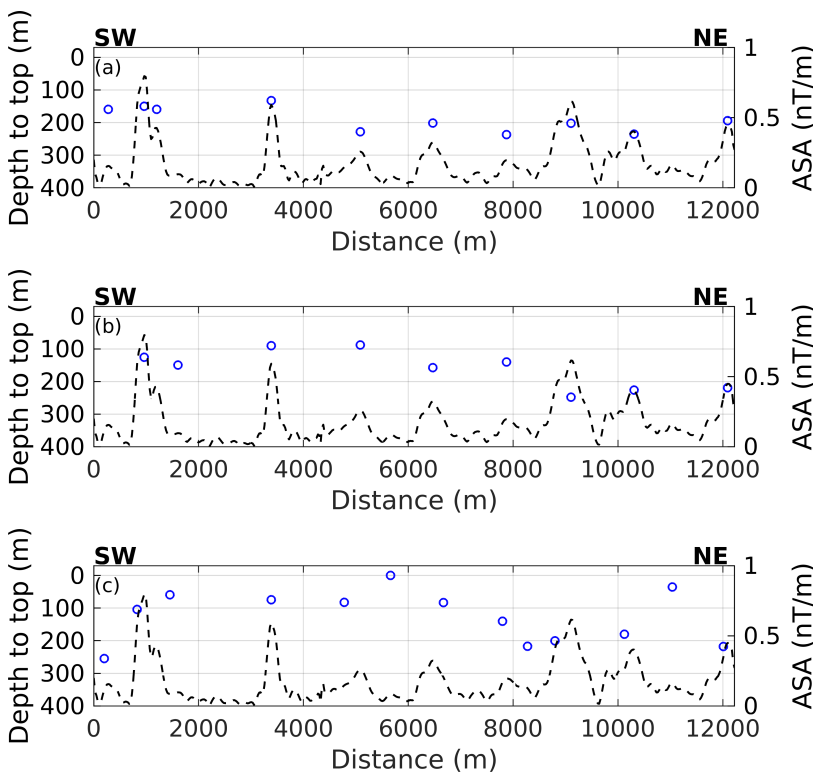

Figure 11: Depth estimates from the curvature method for field data using (a) ASA (data from Fig. 7a), (b) HTD (data from Fig. 8a, red), and (c) RTP (data from Fig. 8a, blue). 


\section{Conclusion}

A new relationship between symmetric and antisymmetric components of a theoretical anomaly profile model has been explored, leading to the reconstruction of the symmetric anomaly through an intuitive transformation of the zero-order analytic signal.

The proposed transformation provides reasonable results for synthetic and field data beyond the well-known case of an isolated anomaly, though the regional field poses difficulties to the reconstruction of the TFA. The field example illustrates the main attributes of the reconstructed VDR: it yields narrower anomalies than the ASA, allowing more accurate location and identification of the sources, and it yields more symmetrical and centralized anomalies in comparison to RTP, because it does not depend on the effective dip angle.

The proposed technique has potential to speed up the interpretation of profile data, as it does not require isolating a single anomaly or a preliminary investigation of remanent magnetism. The method could be applicable to other $2 \mathrm{D}$ models than the dike model.

\section{Acknowledgments}

S. P. Oliveira and F. J. F. Ferreira were financed by CNPq - Ministry of Science, Technology, Innovations, and Communications, Brazil (contracts 313100/2017-9 and 303826/2018-5, respectively).

\section{References}

Bhimasankaram, V. L. S., N. L. Mohan, and S. V. S. Rao, 1978, Interpretation of magnetic anomalies of dikes using Fourier transforms: Geoexploration, 16, 259-266.

Castro, F. R., S. P. Oliveira, J. de Souza, and F. J. F. Ferreira, 2018, Combining tilt derivative filters: new approaches to enhance magnetic anomalies: Brazilian Journal of Geophysics, 36, 1-9.

Castro, L. G., F. J. F. Ferreira, and R. J. Angulo, 2008, Gravimetric-magnetic model of Paranaguá graben, Brazil: Brazilian Journal of Geophysics, 26, 273-292. (In Portuguese).

Cooper, G. R. J., 2015, Using the analytic signal amplitude to determine the location and depth of thin dikes from magnetic data: Geophysics, 80, J1-J6.

de Souza, J., S. P. Oliveira, and F. J. F. Ferreira, 2020, Using parity decomposition for interpreting magnetic anomalies from dikes having arbitrary dip angles, induced and remanent magnetization: Geophysics, 85, J51-J58.

Ferreira, F. J. F., 1982, Aeromagnetic and geological data integration: configuration and tectonic evolution of the Ponta Grossa Arch: Master's thesis, University of São Paulo, Brazil. (In Portuguese).

Hutchison, R. D., 1958, Magnetic analysis by logarithmic curves: Geophysics, 23, 749-769.

Kara, I., M. Özdemir, and F. A. Yüksel, 1996, Interpretation of magnetic anomalies of dikes using correlation factors: Pure and Applied Geophysics, 147, 777-788.

Lessa, G. C., R. J. Angulo, P. C. Giannini, and A. D. de Araujo, 2000, Stratigraphy and Holocene evolution of a regressive barrier in south Brazil: Marine Geology, 165, 87-108.
Louro, V. H. A., A. P. Negrão, L. G. Castro, and F. J. F. Ferreira, 2019, Canoas geophysical anomaly: A possible alkaline body or unusual anomaly caused by mafic dykes in the Ponta Grossa Arch, Brazil?: Journal of Applied Geophysics, 170, 103857.

Murthy, I. V. R., 1985, Magnetic interpretation of dyke anomalies using derivatives: Pure and Applied Geophysics, 123, 232-238.

Nabighian, M. N., 1972, The analytic signal of twodimensional magnetic bodies with polygonal crosssection: its properties and use for automated anomaly interpretation: Geophysics, 37, 507-517.

Paine, J., M. Haederle, and M. Flis, 2001, Using transformed TMI data to invert for remanently magnetised bodies: Exploration Geophysics, 32 , 238-242.

Phillips, J. D., R. O. Hansen, and R. J. Blakely, 2007, The use of curvature in potential-field interpretation: Exploration Geophysics, 38, 111-119.

Pilkington, M., and M. Beiki, 2013, Mitigating remanent magnetization effects in magnetic data using the normalized source strength: Geophysics, 78, J25-J32.

Pirok, B. W. J., S. R. A. Molenaar, L. S. Roca, and P. J. Schoenmakers, 2018, Peak-tracking algorithm for use in automated interpretive method-development tools in liquid chromatography: Analytical Chemistry, 90, 1401114019.

Powell, D. W., 1967, Fitting observed profiles to a magnetized dyke or fault-step model: Geophysical Prospecting, 15, 208-220.

Ram Babu, H. V., and D. Atchuta Rao, 1991, Application of the Hilbert transform for gravity and magnetic interpretation: Pure and Applied Geophysics, 135, 589599.

Rao, B. S. R., and I. V. R. Murthy, 1967, Remanent magnetism of dykes and continental drift: Pure and Applied Geophysics, 68, 124-130.

Reid, A. B., J. M. Allsop, H. Granser, A. J. Millett, and I. W. Somerton, 1990, Magnetic interpretation in three dimensions using Euler deconvolution: Geophysics, 55, 80-91.

Roest, W. R., and M. Pilkington, 1993, Identifying remanent magnetization effects in magnetic data: Geophysics, 58 , 653-659.

Sundararajan, N., P. Srinivasa Rao, and V. Sunitha, 1998, An analytical method to interpret self-potential anomalies caused by 2-D inclined sheets: Geophysics, 63, 1551-1555.

Thompson, D. T., 1982, EULDPH: A new technique for making computer-assisted depth estimates from magnetic data: Geophysics, 47, 31-37. 